\title{
Shape optimization of structural parts in dynamic mechanical systems based on fatigue calculations
}

\author{
P. Häußler, A. Albers
}

\begin{abstract}
In this paper a new method for an automated shape optimization of dynamically loaded components in mechanical systems is presented. The optimization is carried out by means of the results of a durability analysis based on finite elements. Load time histories, which are necessary for durability analyses, are derived from a multibody simulation. The whole optimization loop, which is an iterative procedure, incorporates all these gradual analysis steps and is implemented by the authors in a straightforward, batch-oriented manner using wellknown standard software. Since the whole process involves several different analysis types, such as multibody system simulation and durability analysis, the resulting setup is rather complex. Furthermore, the reader may not be familiar with all the terms arising within the context of every single analysis domain. Therefore, some essential aspects of each of the stages involved in the process are explained to provide the reader with the necessary background. In the following, the required software setup as well as the implementation are described. Finally, an academic example is discussed to illustrate and clearly outline the potential of this method.
\end{abstract}

Key words shape optimization, multibody systems, durability analysis, fatigue, lightweight construction, virtual prototyping

\section{1}

\section{Introduction}

In the past years the significance of computer aided engineering (CAE) methods in the development processes of various products has increased considerably. The strong

Received: 27 October 2003

Revised manuscript received: 1 June 2004

Published online: 27 October 2004

(C) Springer-Verlag 2004

P. Häußler ${ }^{\bowtie}$, A. Albers

IPEK - Institute of Product Development, Department of Mechanical Engineering, University of Karlsruhe, 76128 Karlsruhe, Germany

e-mail: cae@ipek.uni-karlsruhe.de demand for shorter development processes induces the need to reduce physical prototyping and testing by replacing it with virtual prototyping by means of CAE methods.

Finite element methods (FEM) and multibody system simulation (MBS), for example, play a major role in many fields of industrial production, research and development. Especially in the field of structural mechanics, these techniques are established and used extensively.

Although computer-based optimization methods for an application in structural mechanics have been available for several years, they are not very popular. There are several reasons for this:

1. Modelling for computer-based optimization is often complex and time-consuming

2. Optimization results and quality strongly depend on boundary conditions and load cases

3. Optimized models are sometimes difficult to interpret and/or produce in reality

In this paper, points one and two, with regard to the special case of dynamically loaded structural components, which are critical in terms of durability, are addressed. Components in dynamic mechanical systems such as parts of a gear are sometimes subject to time-varying loads, which are often stochastic. When lightweight construction is desired, normally questions concerning durability arise. This is especially true for components where failure of these components leads to safety issues. Therefore, the design process of such components always implies durability investigations such as testing, analytical calculations and/or computer-based durability analysis (DA).

In the research project "Entwicklung und Konstruktion von innovativen Leichtbauprodukten unter konsequenter Verwendung adaptierter Analyse- und Optimierungsmethoden" ${ }^{1}$ (ELAnO) a straightforward methodology for the effective shape optimization of parts for which the issues introduced above are crucial was developed.

The first approaches to consider fatigue behaviour in component design issues were typically based on the fa-

\footnotetext{
1 Development and Design of Innovative Lightweight Structures Applying Adapted Analysis and Optimization Methods
} 
tigue notch factor. Optimization was then carried out in the sense of sizing optimization, i.e., by changing certain geometrical properties such as a bore hole radius. Fanni introduced a concept for the shape optimization of dynamically loaded components in Fanni (1993). Here, a shape optimization scheme directly related to fatigue was developed based on Neuber's theory on fatigue notch factors. Grunwald (Grunwald 1996) and the group of Schnack $^{2}$ developed a new approach for shape optimization based on continuum damage mechanics. For this project, stress redistribution and different cost functions for the mathematical optimization algorithm have been considered.

The authors have contributed to the field of optimization of dynamically loaded components since 1998. In several publications new methods based on coupled simulation processes were presented (Häußler et al. 2001; Müller et al. 1999b,c). A coupled approach for the shape optimization of dynamically loaded components based on results of durability analyses was first presented by Ilzhöfer et al. (2000). Further enhancements of the method and feasibility studies were outlined in Ilzhöfer et al. (2001).

The aim of the research work presented in this paper is to provide a methodology for durability-based shape optimization that is feasible for large real-world applications. The prior investigations mentioned above were limited to small models because of high computational cost due to the usage of mathematical, gradient-based optimization algorithms such as sequential quadratic programming (SQP), for example. Additionally, the identification of suitable design parameters for complex 3D geometries is rather difficult and time-consuming - if possible at all. The research presented here combines an optimality criteria approach with results from durability analyses. Furthermore, this new method takes into account complex load scenarios and load time histories as well as effects on the overall dynamic behaviour of the mechanical system. By incorporating multibody system simulation a complete virtual design optimization process is presented. Moreover, the use of robust standard software packages for the implementation of the new optimization scheme encourages the application by the industry.

\section{1}

\section{The research project ELAnO}

The $\mathrm{ELAnO}^{3}$ project is funded by the Bundesministerium für Bildung und Forschung and was created by a research group consisting of partners from various fields of industry and research dealing with structural optimization.

Nine partners form the following fields take part in ELAnO:

\footnotetext{
2 Prof. Dr.-Ing. habil. E. Schnack, Institut für Technische Mechanik, Universität Karlsruhe

3 See www.elano.org
}

- Research (university):

- IPEK - Institute of Product Development, ${ }^{4}$ University of Karlsruhe

- Institute of Machine Tools and Production Science, University of Karlsruhe

- Chair of Structural Analysis, University of Munich

- Software development (industry):

- FE-Design GmbH

- LMS International

- Application (industry):

- Freudenberg Forschungsdienste

- INDEX-Werke GmbH \& Co. KG Hahn \& Tessky

- TRW Automotive - Global R\&D

- ZF Friedrichshafen AG

The objective of the project is to establish a continuous process chain for the development of lighter, stiffer and more durable products, which can nevertheless be produced at reasonable costs. This will be achieved by the consequent application of numerical methods and interdisciplinary structural optimization and will be evaluated by the development and construction of several lightweight products.

\section{2}

\section{Outline of the optimization process}

The shape optimization of components in dynamic mechanical systems requires several quantities. These quantities are to be derived in every iteration of the optimization process. They result from various types of analyses and the optimization process is obtained by a combination of these analyses. Figure 1 outlines the stages with respect to the order in which they are carried out during the batch process. In the following section some basic aspects of each step of the process shown above are described in more detail in order to provide the reader with the necessary background for all analysis domains involved.

4 Formerly known as "Institute of Machine Design"

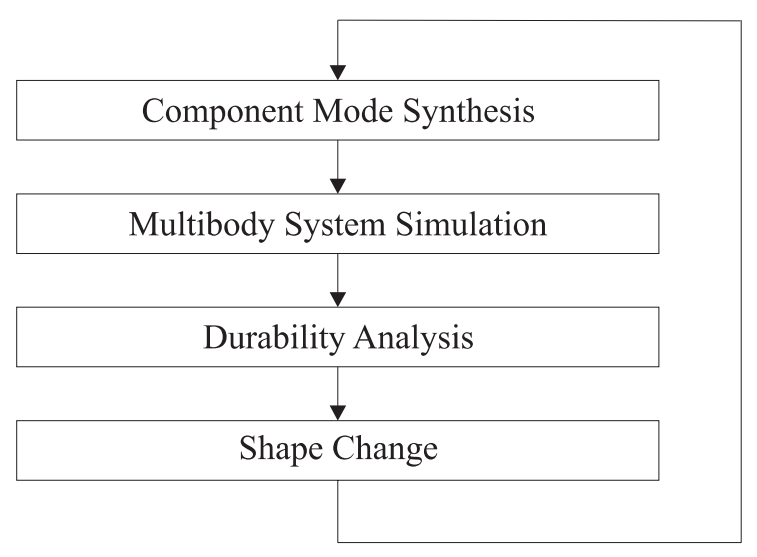

Fig. 1 Stages of the optimization process 
Table 1 Process outline

\begin{tabular}{lll}
\hline Type & Quantity & Software \\
\hline \hline FEM & $\begin{array}{l}\text { Component modes } \\
\text { (vectors and stress tensors) }\end{array}$ & MSC.Nastran \\
MBS & Load time histories & MSC.ADAMS \\
DA & $\begin{array}{l}\text { Stress time histories, } \\
\text { damage distribution }\end{array}$ & FALANCS \\
&
\end{tabular}

\section{3}

\section{Stages and types of analysis}

\section{1}

\section{Flexible multibody systems}

Taking a look at the history of multibody system simulation it becomes evident that the origin of multibody systems were systems of interconnected rigid bodies. Flexibility in those days typically referred to springs and later to linear elastic beams with certain cross sections. The elastic properties of these beams were calculated with the aid of analytical models. As multibody system simulation became more widespread and was applied in a rising number of fields, the need for a more general method for modelling flexible parts - or bodies - arose. The rapidly increasing capabilities of computers formed the basis for dynamic simulations of complex mechanical systems of which flexible parts became more and more crucial.

A very popular approach to meet these demands is well known as the floating frame of reference formulation. The interested reader may consult Shabana (1998) for a comprehensive description.

\subsection{1}

\section{Floating frame of reference formulation}

The idea of this approach is to represent a flexible component in an approximated way by means of a superposition of constant shape functions:

$\bar{u}(t) \approx \sum_{i=1}^{N_{M}} c_{i}(t) \bar{\phi}_{i}$.

Here, the shape $\bar{u}(t)$ of the component at a time instant $t$ is calculated by a weighted sum of time-independent shape functions $\bar{\phi}_{i}$. The time dependence is contained entirely in the scalar weighting factors $c_{i}(t)$. The benefit of this approach is a significant reduction of the degrees of freedom necessary to describe the flexibility of the component. ${ }^{5}$

In order to justify the approach mentioned in (1), a fundamental assumption is required. The deformations

\footnotetext{
5 All quantities expressed in a local, body-fixed coordinate system are marked with a bar.
}

of the flexible components are considered to be small with regard to a local, i.e., a body-fixed system of coordinates, which means that geometric non-linearity must be negligible. Moreover, they are not to exceed the elastic limit of the material used.

In this method, elastic deformations are expressed within the body-fixed system of coordinates. The gross body motion within space is captured by six rigid body degrees of freedom, namely three translational and three rotational coordinates. The shape functions $\bar{\phi}_{i}$ are also expressed in the body fixed coordinate system.

By means of the finite element method a very effective and straightforward implementation of the floating frame of reference formulation can be achieved.

\subsection{2}

\section{Component mode synthesis}

In the previous section an approximation for the component deformation was introduced by means of a weighted sum of constant shape functions. When dealing with the task of deriving these functions the finite element method can be very effective. Here, a well-known and widely used concept known as component mode synthesis (CMS) can be used. One of the most common approaches (Craig and Bampton 1968) is based on the idea of using normal mode analysis techniques to calculate eigenvectors for use as shape functions, or shape vectors, respectively. While employing eigenvectors for approximation was already very widespread, Craig and Bampton among others (Hurty 1965) enhanced the method by taking into account additional types of vectors. In the following, Craig and Bampton's method is dealt with in more detail since it is also implemented in MSC.ADAMS/Flex. Here, the following types of vectors, or modes are utilized:

1. Fixed boundary normal modes

2. Static correction modes

Fixed boundary normal modes are eigenvectors that result from a finite element normal mode analysis. They are connected with the boundary condition implying that all nodes of the finite element model are fixed at which forces and joints that are applied within the multibody system. In the following sections, these nodes are referred to as interface nodes.

Static correction modes are deformation vectors that result from static load cases with which loads are applied to interface points. Typically, a unit load is applied to every nodal coordinate, whereas all other interface nodes are fixed. This leads to six static correction modes for each interface node. Figure 2 illustrates some mode shapes for a one-dimensional bar. The shapes (a) and (b) are fixed-boundary normal modes, shapes (c) and (d) are static correction modes resulting from a unit displacement (c) and a unit rotation (d), respectively. The use of static correction modes ensures a good approximation of the deformation when forces and moments are 


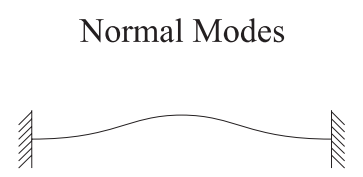

(a)

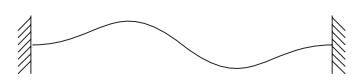

(b)
Static Correction Modes

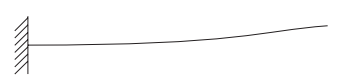

(c)

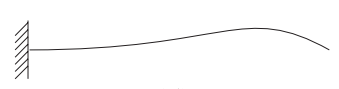

(d)
Fig. 2 Mode shape examples for a one-dimentional bar

applied to interface points. The fixed boundary normal modes are important as soon as high frequency excitation is expected, i.e., if the loading may not be considered "quasi-static".

Note: In the following, the flexible component is always assumed to be represented by a finite element model.

\subsection{3}

\section{Dynamic equation of motion and modal coordinates}

The dynamic equation of motion for a linear elastic structural component is as follows:

$\overline{\mathbf{M}} \ddot{\overline{\mathbf{u}}}(t)+\overline{\mathbf{D}} \dot{\overline{\mathbf{u}}}(t)+\overline{\mathbf{K}} \overline{\mathbf{u}}(t)=\overline{\mathbf{F}}(t)$.

Here $\overline{\mathbf{u}}(t)$ denotes the time-dependent vector of displacements of nodal coordinates. $\overline{\mathbf{M}}$ is the finite element mass matrix, $\overline{\mathbf{D}}$ a damping matrix and $\overline{\mathbf{K}}$ the stiffness matrix, respectively.

Inserting the approximation approach (1) leads to:

$\overline{\mathbf{M}} \sum_{i=1}^{N_{M}} \ddot{c}_{i}(t) \bar{\phi}_{i}+\overline{\mathbf{D}} \sum_{i=1}^{N_{M}} \dot{c}_{i}(t) \bar{\phi}_{i}+\overline{\mathbf{K}} \sum_{i=1}^{N_{M}} c_{i}(t) \bar{\phi}_{i}=\overline{\mathbf{F}}(t)$.

Equation (3) represents a coupled system of $N_{M}$ differential equations. The whole time dependence is contained in the weighting factors or modal amplitudes $c_{i}(t)$. Note that the mode shapes $\bar{\phi}_{i}$ are constant.

The equations in (3) are generally coupled for two reasons:

1. Static correction mode shapes and eigenvectors are generally not perpendicular in pairs, i.e., $\bar{\phi}_{i} \cdot \bar{\phi}_{j} \neq 0$

2. The matrices $\overline{\mathbf{M}}, \overline{\mathbf{K}}, \overline{\mathbf{D}}$ typically have no diagonal structure

Concerning the first point mentioned above, an eigenvalue analysis on the system of equations described in (3) is to be carried out. In order to deal with the second point, modal damping can be employed. In this context, it is very convenient to first neglect damping and introduce modal damping sometime later.

The eigenvalue analysis for the undamped system is obtained by solving the well-known equation:

$\left(\overline{\mathbf{K}}-\overline{\mathbf{M}} \omega^{2}\right)=0$.
This leads to a new set of eigenvectors $\bar{\phi}_{i}^{*}$ with associated eigenvalues $\lambda_{i}^{*}=\omega^{2}$ :

$\bar{\phi}_{i}^{*}, \lambda_{i}^{*} \quad i \in\left[1 \cdots N_{M}\right]$.

Due to the very nature of eigenvectors this new set of vectors is indeed an orthogonal basis, which implies that the following conditions hold:

$\bar{\phi}_{i}^{*} \cdot \bar{\phi}_{j}^{*}=0 \quad: \quad i \neq j$,

$\bar{\phi}_{i}^{*} \cdot \bar{\phi}_{j}^{*} \neq 0 \quad: \quad i=j$.

The vectors $\bar{\phi}_{i}^{*}$ form an orthogonal basis of the same space like the vectors $\bar{\phi}_{i}$. With this new set of shape vectors the dynamic equation of motion (3) can now be written in the following form (damping neglected):

$\overline{\mathbf{M}} \ddot{\mathbf{c}}^{*}(t) \overline{\boldsymbol{\Phi}}^{*}+\overline{\mathbf{K}} \mathbf{c}^{*}(t) \overline{\boldsymbol{\Phi}}^{*}=\overline{\mathbf{F}}(t)$.

Here the sums in (3) are replaced by introducing the modal shape matrix $\overline{\boldsymbol{\Phi}}^{*}$ and a vector of new modal coordinates $c_{i}^{*}$ :

$\overline{\mathbf{\Phi}}^{*}=\left[\bar{\phi}_{1}^{*}, \bar{\phi}_{2}^{*}, \cdots, \bar{\phi}_{N_{M}}^{*}\right]$,

$\mathbf{c}^{*}=\left[c_{1}^{*}, c_{2}^{*}, \cdots, c_{N_{M}}^{*}\right]^{T}$.

Multiplying (7) with the transpose of the modal shape matrix results in:

$\ddot{\mathbf{c}}^{*}(t) \overline{\boldsymbol{\Phi}}^{*^{T}} \overline{\mathbf{M}} \overline{\boldsymbol{\Phi}}^{*}+\mathbf{c}^{*}(t) \overline{\boldsymbol{\Phi}}^{*^{T}} \overline{\mathbf{K}} \overline{\boldsymbol{\Phi}}^{*}=\overline{\boldsymbol{\Phi}}^{*^{T}} \overline{\mathbf{F}}(t)$.

In (9) one can clearly see that the single equations in the system are now decoupled due to the orthogonality property of the shape vectors. Thus (9) can be written as a set of $N_{M}$ independent differential equations in the modal coordinates $c_{i}^{*}$ :

$$
\begin{array}{ccc}
\ddot{c}_{1}^{*}(t) \bar{m}_{1}+c_{1}^{*}(t) \bar{k}_{1} & =\bar{f}_{1}(t) \\
\ddot{c}_{2}^{*}(t) \bar{m}_{2}+c_{2}^{*}(t) \bar{k}_{2} & =\bar{f}_{2}(t) \\
\vdots & = & \vdots \\
\ddot{c}_{N_{M}}^{*}(t) \bar{m}_{N_{M}}+c_{N_{M}}^{*}(t) \bar{k}_{N_{M}} & =\bar{f}_{N_{M}}(t)
\end{array}
$$

Here, the right side of the equation contains the generalized modal forces $\bar{f}_{i}$, which are defined as follows:

$\overline{\mathbf{f}}=\overline{\boldsymbol{\Phi}}^{* T} \overline{\mathbf{F}}$.

On the left side, the generalized modal mass and stiffness can be recognized:

$\bar{m}_{i}=\bar{\phi}_{i}^{* T} \overline{\mathbf{M}} \bar{\phi}_{i}^{*}=1 \quad \bar{k}_{i}=\bar{\phi}_{i}^{* T} \overline{\mathbf{K}} \bar{\phi}_{i}^{*}$

The fact that the generalized modal mass equals one is due to the fact that vectors are expected to be normalized 
with respect to their generalized mass since this is common in finite element analysis. With the approximation approach outlined above, the degrees of freedom of the finite element model representing the flexible part are considerably reduced based on component mode synthesis. In fact, the number of degrees of freedom does not depend on the finite element mesh, i.e., the number of nodes, at all. It is rather the number of modes within the modal basis that defines the number of dynamic equations. Here, it becomes evident that the number of interface nodes is critical since every additional interface node causes six additional modes.

Damping can be included in the equations mentioned in (10) by means of modal damping in every single equation by a modal damping coefficient $\bar{d}_{i}^{*}$ :

$\ddot{c}_{i}^{*}(t) \bar{m}_{i}+\dot{c}_{i}^{*}(t) \bar{d}_{i}^{*}+c_{i}^{*}(t) \bar{k}_{i}=\bar{f}_{i}(t)$.

The deformation of the flexible component at a time instant $t$ can now be calculated from the values of the modal coordinates $c_{i}^{*}(t)$ together with the constant precomputed shape vectors $\bar{\phi}_{i}^{*}$. In the following, it will be demonstrated that by means of modal coordinates and pre-computed modal stress tensors stress distributions can also be calculated.

\section{2}

\section{Durability analysis}

In computer-aided, finite-element-based durability or fatigue analysis there are two major approaches ${ }^{6}$ (Bannantine et al. 1990):

\section{- Stress-life or S-N approach \\ - Strain-life approach}

Due to the assumption that the deformations of the flexible part are small and do not exceed the elastic limit of the material, the stress-life approach is employed in the following. Local plastic strain components are assumed not to be of importance or, in other words, even local stresses do not exceed the elastic limit of the material.

\subsection{1}

\section{Stress-life approach}

The stress-life approach is well suited for problems that fall into a category known as high-cycle fatigue (HCF). Failure should not occur until $10^{4}$ load cycles are completed (Bishop and Sherratt 2000).

The basic concept of the stress-life approach is the material-related S-N curve or "Wöhler's curve", which relates stresses to the number of cycles until crack initiation. In the stress-life approach crack initiation is typically treated as failure.

\footnotetext{
${ }^{6}$ Concepts dealing with crack propagation are not taken into account
}

The basic procedure to determine a damage distribution consists of the following steps:

1. Generate local stress time histories

2. Classify local stress time histories, typically by methods such as rain flow cycle counting

3. Accumulate partial damages of each class for every point (node) on the surface of the component considered

The durability analysis code FALANCS of LMS International used for the work described in this paper enhances this basic procedure substantially and includes sophisticated methodologies for state-of-the-art fatigue prediction such as load time histories, filtering and a powerful critical plane approach. For a detailed description of the theory and capabilities the FALANCS theory manual is to be consulted (LMS International 2002).

\section{2 .2}

\section{Stress approximation by modal superposition}

Stress time histories needed for the damage calculation can be easily and efficiently calculated from modal coordinate time series and modal stress tensors. During the finite element analysis required for the component mode synthesis, stress tensors can be calculated in a straightforward manner and stored in an output database. Every single one of these stress tensors is associated with one of the final mode vectors.

During the dynamic multibody system simulation time series for modal coordinates $c_{i}^{*}$ are computed. With these two quantities, stress time histories can be calculated as follows:

$\bar{\sigma}(t)=\sum_{i=1}^{N_{M}} c_{i}^{*}(t) \cdot \overline{\mathbf{S}}_{i}=\overline{\mathbf{c}}^{*}(t) \cdot \overline{\mathbf{S}}$.

Here, the matrix $\overline{\mathbf{S}}$ contains all modal stress tensors. The use of this method for all time steps may result in a huge amount of data. In state-of-the-art software packages such as FALANCS sophisticated signal processing procedures are carried out on the modal coordinate time series prior to calculating stress time histories as formulated in (14) in order to significantly reduce both the amount of data and computation time (LMS International 2002).

Since a durability analysis is to be carried out based on the calculated stresses the quality of the latter is crucial. An error in local stress results of, e.g., 10\% can lead to an error in the durability results of about $100 \%$ ! The source of this effect is obviously a material property, namely the non-linear S-N curve. Therefore, the choice of a sufficient modal basis is highly significant, especially when high frequency loading is expected.

In the methodology described in this paper all stresstime history and damage calculations are carried out by means of FALANCS. 


\section{3}

\section{Parameter-free shape optimization}

\subsection{1}

\section{Optimality criteria methods}

In contrast to the mathematical programming methods, the optimality criteria methods take advantage of the knowledge about the physics and mechanics of the respective problem set. Theses will be postulated describing the optimum.

A well-known and ascertained physical law relating to structural mechanics is, e.g., the fully stressed design, which can only be applied to statically determined structures. An important mathematical optimality criterion is the Karush-Kuhn-Tucker condition (Karush 1939; Kuhn and Tucker 1951) normally designed for convex optimization purposes.

The theses on stress homogenization and stress minimization can also be considered as optimality criteria (Schnack 1978). Regarding the optimality criteria methods, these criteria and the response behaviour of modifications of the physical model are implemented into the algorithm. With suitable redesign rules, a convergence behaviour is achieved that cannot be attained with mathematical optimizers. Applying this particular physical and mechanical knowledge, the optimality criteria methods remain limited to the certain application areas.

The optimality criteria are particularly well proven for shape and topology optimization where a large number of design variables is required. It is important to note that the convergence speed is independent of the number of design variables.

\subsection{2 \\ TOSCA.shape}

The optimization program TOSCA used in the method described in this paper is based on the code computeraided optimization system Sauter (CAOSS), which is also the basis of MSC.Construct (Allinger et al. 1996; Sauter 1992; Müller et al. 1999a). The software was developed by FE-Design 7 , a spin-off company of the Institute of Product Development (IPEK) at the University of Karlsruhe. The authors contribute to the further development of this code that has proven feasibility and efficiency in various industrial applications throughout the past years.

TOSCA.shape implements an optimality criteria approach. A distribution of scalar values within a design area, the so-called design response are basically processed by the optimization algorithm. Depending on the userdefined objective function these values are minimized or maximized by means of a homogenization within the design area. The shape change induced by the optimizer, i.e., the variation of the finite element mesh is managed by

\footnotetext{
7 www.fe-design.de
}

displacing nodes. This leads to a decrease of the local surface curvature in highly stressed regions. Typically such scalar quantities are von Mises stresses obtained from a static finite element analysis. The nodal displacement $\Delta U$ that is applied to a certain node by the optimizer is then calculated by means of the following formula:

$\Delta U_{n}=\alpha \cdot F(a, b, \cdots)$.

Here $\alpha$ denotes a scalar calibration factor and $F(a, b, \cdots)$ is a function of the scalar nodal design response $a$ used for optimization such as von Mises stress, a reference value $b$ and other parameters.

In the following sections, a method for nodal displacement based on quantities from durability analyses is presented.

\section{4}

\section{Optimization based on damage distribution}

\subsection{1 \\ Review of the concept}

Initial IPEK research on shape optimization with regard to durability resulted in two types of quantities that were obtained from a durability analysis (MSC.Fatigue) for the use as design response (Ilzhöfer et al. 2000):

1. Number of cycles to failure $D_{\text {Resp. }}=N$

2. Logarithmic damage values $D_{\text {Resp. }}=\log _{10}(D)$

The use of logarithmic values instead of real damage values is common practice due to the logarithmic nature of fatigue. In order to avoid changes to the algorithm implemented in TOSCA.shape, ${ }^{8}$ these values were used directly as input to the controller of TOSCA.shape that is at the same place where stresses are usually used (see (15)). This was done in order to keep the code of TOSCA.shape as simple as possible (one algorithm for both, stress- and damage-based shape optimization).

This early research has demonstrated the potential of the method, but it has also shown that the algorithm in TOSCA.shape was not suitable for both chosen types of quantities, cycles to failure and logarithmic damage values. The reason for this is the difference in the gradients that can be observed when comparing the latter of typical stress and cycles to failure/logarithmic damage distributions. Compared to stress gradients, cycles to failure distributions show a very high gradient where the gradient of logarithmic damage values is typically very small.

To avoid modifications to the algorithm of the optimizer a new approach was developed in order to overcome

\footnotetext{
8 In the past the optimization software was distributed by MSC.Software and labeled "MSC.Construct". The code is basically the same as TOSCA.shape. To avoid confusion in this paper, the term "TOSCA" will also be used if referring to past research that was actually carried out with MSC.Construct.
} 
the issues related to the gradients. The basic concept is to calculate a related damage equivalent stress amplitude from nodal damage values (Ilzhöfer et al. 2001). This results in gradients that can be compared to those of stresses resulting from regular finite element calculations. It is important to keep in mind that this distribution calculated from a damage distribution may in general be something completely different from the stress distributions resulting from finite element analyses with certain load cases. High stress values will occur at locations of high damage. These locations may differ from the locations of the highest stresses observed when looking at finite element results from single load cases. The reasons for this are certainly the load time histories and the fatigue behaviour of the material.

In early investigations (Ilzhöfer et al. 2001) the related stress distribution was calculated from durability analysis results (namely cycles to failure $N_{f}$ ) using the MansonCoffin-Morrow relationship:

$\epsilon_{t}=\underbrace{\frac{\sigma_{f}^{\prime}}{E}\left(2 N_{f}\right)^{b}}_{\text {elastic strain }}+\underbrace{\epsilon_{f}^{\prime}\left(2 N_{f}\right)^{c}}_{\text {plastic strain }}$.

In this equation the total strain is expressed as the sum of elastic and plastic strain components, $\sigma_{f}^{\prime}$ denotes the fatigue strength coefficient, $\epsilon_{f}^{\prime}$ is the fatigue ductility coefficient and $E$ the elastic modulus of the material, respectively. The fatigue strength exponent $b$ is also a material dependent parameter and usually varies between -0.05 and -0.12 (Bannantine et al. 1990).

By only assuming elastic stresses and strains, the second term in (16) can be neglected. Therefore, a damagerelated stress amplitude can be defined as follows:

$\sigma_{e q}=\sigma_{f}^{\prime}\left(2 N_{f}\right)^{b}$

Using the damage-related stress distribution as input for TOSCA.shape, it leads to a feasible method for the shape optimization of parts with respect to durability. The problems related to gradients are solved and the design response now used - the damage related equivalent stress - still contains all effects of load time histories and material properties. Especially the spatial properties of the damage distribution are retained in the distribution of the damage related equivalent stress.

\subsection{2}

\section{TOSCA.shape and FALANCS interfacing}

The example presented in this paper is based on an interfacing of TOSCA.shape and the durability analysis code FALANCS from LMS International. In the scope of the ELAnO project, an interface has been developed that is based on the concepts of the approach described above using damage related stress distributions as a design response.
First a design life $N_{\text {Design }}$ is specified by the user. This value represents the intended number of successively endured repetitions of the respective load time histories. During the durability analysis, the actual life $N$, or the actual number of endured repetitions, is calculated by FALANCS. Related stress amplitudes $\sigma$ and $\sigma_{\text {Design }}$ are derived for both values by using the S-N curve. When a strain life approach is used in FALANCS, a synthetically generated S-N curve is used in this procedure. This $\mathrm{S}-\mathrm{N}$ curve is derived when using the well-known laws of Manson-Coffin-Morrow and Ramberg-Osgood, as well as Neuber's formula.

As input for the optimizer of TOSCA.shape - i.e., as a design response - the quotient of the related stress amplitudes is used as follows:

$D_{\text {Resp. }}=\frac{\sigma}{\sigma_{\text {Design }}}$.

Optimizing the component's life means to minimize the design response defined in (18). This is achieved by the well approved homogenization technique that is implemented in TOSCA.shape. This technique is also used for shape optimization based on usual stress distributions that are derived from static finite element analysis. Figure 3 summarizes the TOSCA.shape-FALANCS interfacing. The damage calculation in FALANCS requires stresses and load time histories. Due to the modal superposition technique used here, modal stress tensors from the component mode synthesis analysis with MSC.Nastran are used. Therefore, the respective load time histories are modal coordinate time histories. These may be exported directly from a multibody system code like MSC.ADAMS.

\section{FALANCS}

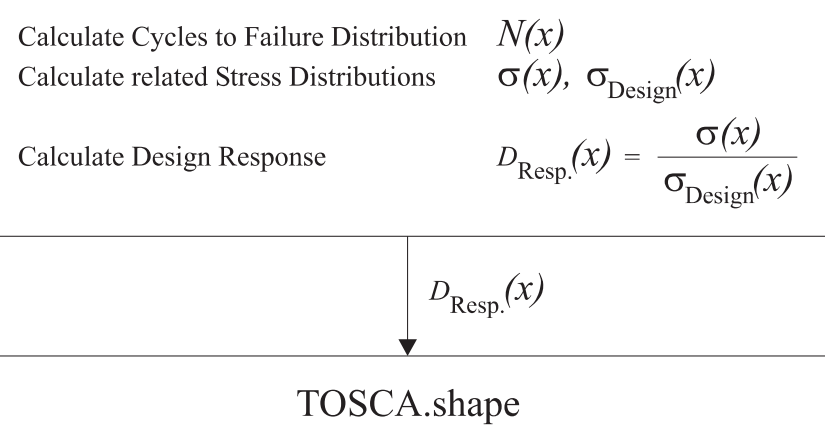

Fig. 3 TOSCA.shape-FALANCS interfacing

Table 2 Material properties

\begin{tabular}{lc}
\hline Property & Value \\
\hline \hline Endurance limit $\sigma_{E}$ & $4 \times 10^{7}[\mathrm{~Pa}]$ \\
Tensile strength $\sigma_{\max }$ & $5 \times 10^{8}[\mathrm{~Pa}]$ \\
Pressure resistance $\sigma_{P}$ & $1 \times 10^{9}[\mathrm{~Pa}]$ \\
\hline
\end{tabular}


In addition to modal stress information and modal coordinate time series, a material model (S-N curve) has to be selected from a database or explicitly specified by the user. Table 2 summarizes the values used in the example discussed below.

It is important to keep in mind that there are several major differences in the new process described in this paper to the earlier research mentioned above:

- There is no need for measured load time histories due to the use of modal coordinate time series from MBS simulation. Arbitrary complex time histories and load scenarios may be used

- It is not limited to pure elastic damage models as shown in (17)

- It is a feasible process for large models of real world applications due to efficient parameter-free optimization algorithms and the use of robust standard software packages

- Updates of modal coordinate time series in every iteration of the optimization process. Full coverage of effects on the mechanical system as a whole

- The design optimization process is completely covered by means of simulation

\section{4}

\section{The optimization process}

The important aspects of all analysis techniques involved in the optimization process were discussed in the previous sections. In this section a brief summary and overview of the process as a whole is presented. Figure 4 illustrates the subsequent steps and dataflow within one single iteration of the optimization.

If the component mode synthesis is carried out with MSC.Nastran the mode shape vectors $\phi_{\text {Modal }}^{i}$ and modal

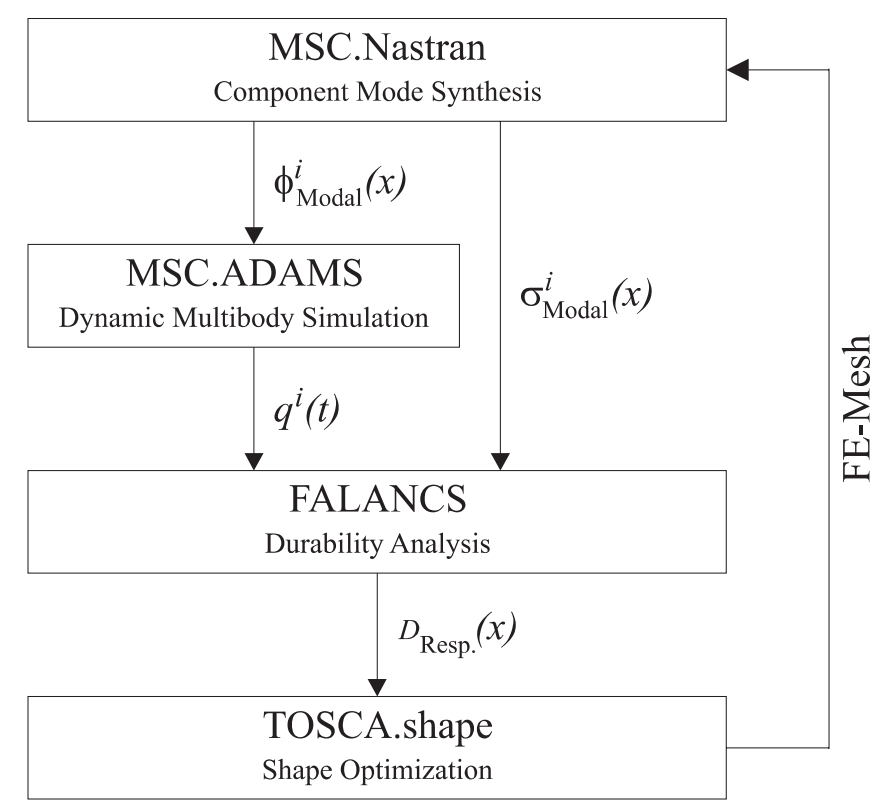

Fig. 4 Overview of the optimization process stress tensors $\sigma_{\text {Modal }}^{i}$ are obtained. The index $i$ refers to the mode number. Using the mode shape vectors a dynamic multibody system simulation is performed using MSC.ADAMS. Modal coordinate time series $q^{i}(t)$ are exported from this analysis. Together with modal stress tensors, these time series are transferred to FALANCS for a durability analysis that is based on a previously user-defined material model and method parameters. Finally, the intended design response $D_{\text {Resp. }}(x)$, i.e., a stress quotient distribution on the component's surface as defined in (18) is derived and provided to the optimizer TOSCA.shape. Following the optimization the modified finite element mesh is then again transferred to MSC.Nastran for a new component-mode synthesis.

The handling of data files and the initialization of every individual analysis step is carried out completely by the control program of TOSCA.shape. Once the process has been started, no further user action is required.

\section{5}

\section{Example: plate with hole}

The new method will be demonstrated using a simple academic example in this section. Figure 5 shows a square plate with a hole in its centre. The plate will be used as flexible part within a multibody system where it will be subject to certain loading conditions. The shape optimization will be carried out on the border of the hole, i.e., the optimization code will change the shape of the hole to increase the overall lifetime of the plate.

\section{1}

\section{Finite element model}

The plate is meshed with 4031 elements of type MSC.Nastran CQUAD4 (quadrilateral plate element: isoparametric membrane-bending or plane strain (Schaeffer 2001)). In this example the shell properties for the elements (MSC.Nastran PSHELL) have been defined to support membrane, bending and transverse shear behaviour.

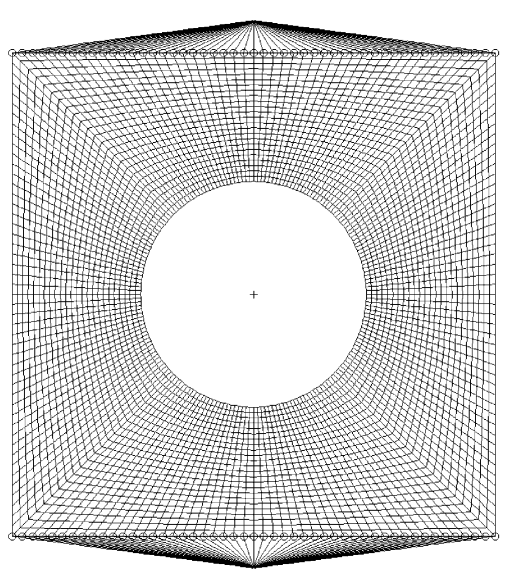

Fig. 5 Plate with hole 
Moreover, there are two additional nodes at the centre of two opposite borders of the plate with a $10 \mathrm{~mm}$ offset. These nodes are used as interface nodes, i.e., as nodes to which forces or joints are applied in the multibody system. The plate itself is connected to these interface nodes by rigid body elements of type MSC.Nastran RBE2. The nodes on each border next to an interface node are connected with these RBE2 elements via the associated interface node as illustrated in Fig. 6. In this example, the circle refers to the position of the interface node. This way it is possible to fix the plate border by constraining all degrees of freedom of the respective interface point.

The component mode synthesis was carried out with MSC.Nastran. Tables 3 and 4 contain information on the modal basis. The first six modes (highlighted in Table 3) can be easily identified as rigid body modes by considering the associated eigenfrequencies, which are below $1 \times 10^{-3} \mathrm{~Hz}$. These modes are removed from the set of basis vectors within MSC.ADAMS. Rigid body motion is -

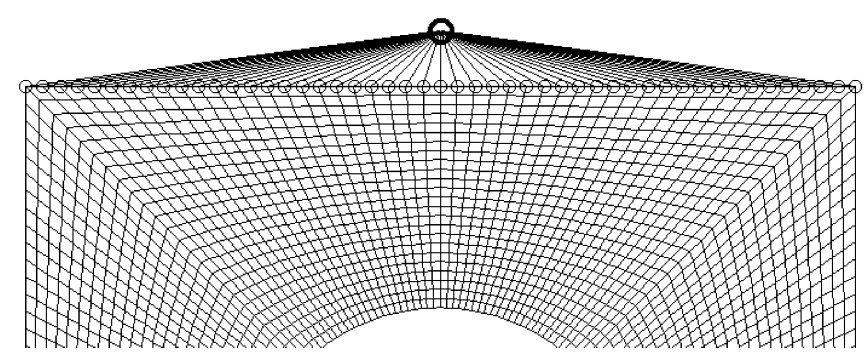

Fig. 6 Finite element mesh and RBE2 element

Table 3 Mode types and mode count

\begin{tabular}{ll}
\hline Number of eigenmodes & 20 \\
Number of static correction modes & 12 \\
Total number of modes in basis & $\mathbf{3 2}$ \\
\hline
\end{tabular}

Table 4 Eigenfrequencies of the orthogonal modes

\begin{tabular}{rlll}
\hline No. & Freq. [Hz] & No. & Freq. [Hz] \\
\hline \hline 1 & $\mathbf{1 . 8 3} \times \mathbf{1 0}^{-\mathbf{3}}$ & 17 & $1.51 \times 10^{3}$ \\
2 & $\mathbf{1 . 5 9} \times \mathbf{1 0}^{-\mathbf{3}}$ & 18 & $1.80 \times 10^{3}$ \\
3 & $\mathbf{1 . 2 2} \times \mathbf{1 0}^{-\mathbf{3}}$ & 19 & $1.85 \times 10^{3}$ \\
4 & $\mathbf{5 . 7 1} \times \mathbf{1 0}^{-\mathbf{4}}$ & 20 & $2.01 \times 10^{3}$ \\
5 & $\mathbf{6 . 7 7} \times \mathbf{1 0}^{-\mathbf{4}}$ & 21 & $2.25 \times 10^{3}$ \\
6 & $\mathbf{8 . 6 7} \times \mathbf{1 0}^{-\mathbf{4}}$ & 22 & $2.39 \times 10^{3}$ \\
7 & $1.58 \times 10^{2}$ & 23 & $2.65 \times 10^{3}$ \\
8 & $2.07 \times 10^{2}$ & 24 & $2.74 \times 10^{3}$ \\
9 & $4.89 \times 10^{2}$ & 25 & $2.88 \times 10^{3}$ \\
10 & $5.67 \times 10^{2}$ & 26 & $2.91 \times 10^{3}$ \\
11 & $5.93 \times 10^{2}$ & 27 & $3.00 \times 10^{3}$ \\
12 & $7.87 \times 10^{2}$ & 28 & $5.95 \times 10^{3}$ \\
13 & $9.33 \times 10^{2}$ & 29 & $8.18 \times 10^{3}$ \\
14 & $1.01 \times 10^{3}$ & 30 & $8.90 \times 10^{3}$ \\
15 & $1.25 \times 10^{3}$ & 31 & $1.27 \times 10^{4}$ \\
16 & $1.27 \times 10^{3}$ & 32 & $1.70 \times 10^{4}$ \\
\hline
\end{tabular}

as pointed out earlier - then realized by adding six degrees of freedom to the local body coordinate system.

\section{2 \\ Multibody system}

After the plate finite element model and a MSC.Nastran component mode synthesis is prepared the plate can be imported into MSC.ADAMS. Figure 7 shows a schematic drawing of the multibody system that was used for the research presented here. In Fig. 8 the MSC.ADAMS model is depicted. The system consists of four bodies (plate, two links and ground) and therefore is simple but completely fulfils its purpose. The plate is connected to the ground by a fixed joint at one of its interface nodes. The fixed joint (symbolized by the lock-icon) therefore locks all degrees of freedom of that point.

Motion is introduced by a time-variable enforced displacement that is applied to the translational joint of the upper link. The two links are connected by a revolute joint as is the lower link and the plate at the opposite interface point. They act as a type of crank mechanism and result in an enforced displacement in a direction along the plate normal. Two links are necessary, since applying an enforced displacement to only one link or directly to the plate led to high stresses within the plate's $x y$-plane. The translational joint applied to the upper link ensures pure motion of this link along the global $z$-axis.

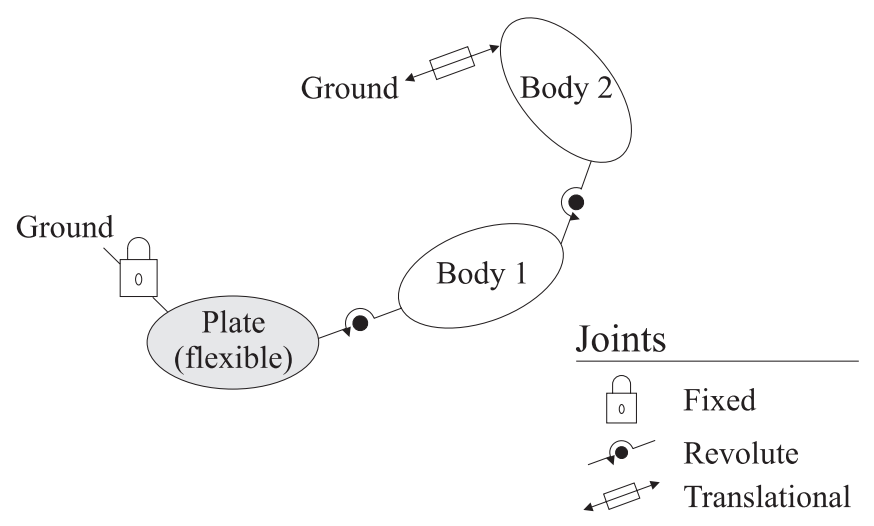

Fig. 7 Multibody system - schematic

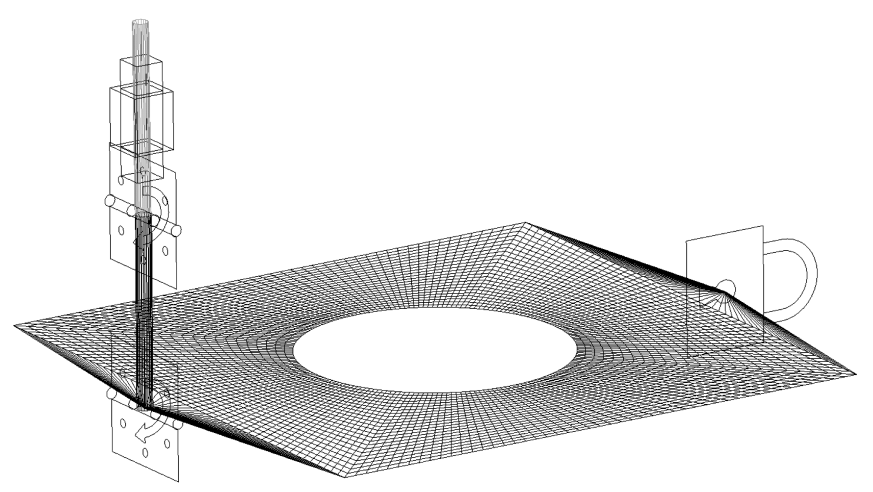

Fig. 8 Multibody system - MSC.ADAMS 
Table 5 Enforced displacements

\begin{tabular}{lll}
\hline No. & $d_{z}(t)$ & Values \\
\hline \hline 1 & $A_{1} \cdot \sin \left(\omega_{1} t\right)$ & $A_{1}=4 \mathrm{~mm}$ \\
& & $\omega_{1}=2 \pi$ \\
2 & $A_{2} \cdot \sin \left(\omega_{2} t\right)$ & $A_{2}=0.5 \mathrm{~mm}$ \\
& & $\omega_{2}=1468$ \\
3 & $A_{3} \cdot \sin \left(\omega_{1} t\right)+A_{4} \cdot \sin \left(\omega_{2} t\right)$ & $A_{3}=3.5 \mathrm{~mm}$ \\
& & $A_{4}=0.25 \mathrm{~mm}$ \\
\hline
\end{tabular}

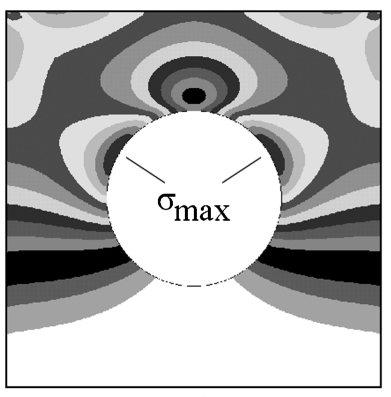

(a)

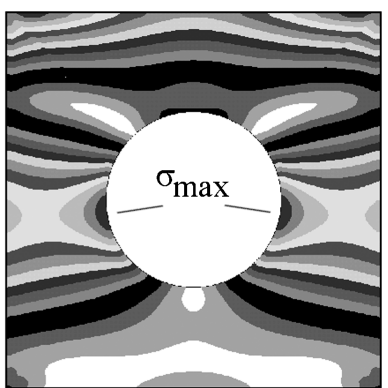

(b)
Fig. 9 Von Mises stress distribution

Three different dynamic simulations have been carried out, each with different enforced displacements $d_{z}$ of the upper link. Details about the excitation are presented in Table 5).

Simulation one is based on a $1 \mathrm{~Hz}$ sinusoidal enforced displacement. The excitation frequency is well below the first eigenfrequency of the one-sided fixed plate. The displacement result for the plate can be seen as quasi-static. Simulation two is based on a $233 \mathrm{~Hz}$ sinusoidal excitation. In this simulation, the third eigenmode of the onesided fixed plate is excited. Finally the third simulation uses a linear combination of the two excitations described before.

The frequencies were chosen after looking at von Mises stress distributions for a static deflection of the plate and the stress distributions of the eigenmodes. The goal was to select two stress distributions wherein the location of the highest stress level on the border of the hole differs. Figure 9 shows von Mises stresses for a static deflection (a) and the stress distribution associated with the $233 \mathrm{~Hz}$ eigenmode (b). The different locations of the highest stresses can be observed clearly. The maximum Amplitudes $A_{i}$ used in the simulations were adjusted in such a way that resulting damages in all three cases were within the same order of magnitude.

\section{3}

\section{Optimization model}

The biggest possible advantage of a parameter-free shape optimization is the simple and straightforward procedure to set up everything that is necessary to define the optimization problem. Since everything is carried out on the

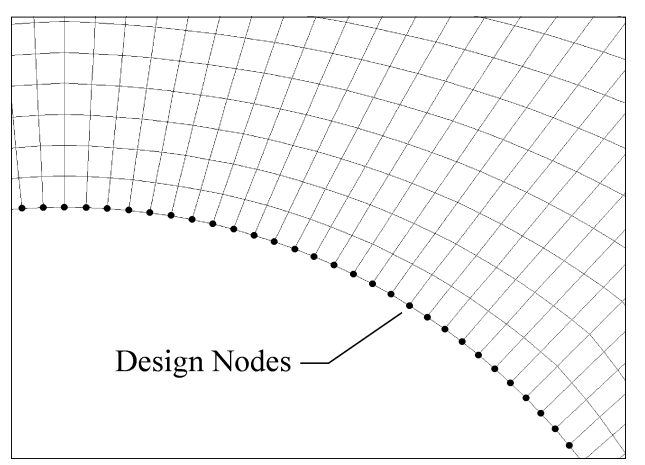

Fig. 10 Design nodes on the border of the hole

finite element mesh, no parameterized geometry is necessary. Furthermore, shape basis vectors commonly used for shape optimization are not needed with TOSCA.shape. Nodal degrees of freedom are directly used as design variables instead.

In the problem presented here, the aim is to find an optimal shape for the hole in the plate's centre. Therefore, only the nodes on the border of the hole as depicted in Fig. 10 are used as design variables. The objective function can be simply stated as:

\section{"Minimize the damage related stress distribution within the design area."}

In TOSCA.shape this is achieved by a homogenization of the design response throughout the design area, that is on the border of the hole. It is important to note that during the iterations the shape of the hole will change and may no longer be a circle. ${ }^{9}$

The iterative process ends when a user defined stop condition is fulfilled. This may be a maximum allowed equivalent stress value or simply a maximum number of iterations.

\section{4 \\ Results}

\subsection{1}

\section{Shape of the hole}

Figure 11 shows the location of the maximum damage $D_{\text {max }}$ for the starting design with respect to all three different excitations. As expected, the maximum is always on the border of the hole. The actual position varies in all of the three models due to the different dynamic excitations which in turn lead to a different dynamic stress tensor in the plate. When the two excitations $(1 \mathrm{~Hz}$ and $233 \mathrm{~Hz}$ ) are superposed, the maximum is between the maxima of the single frequency models.

Optimization results for the three models are shown in Fig. 12. The resulting optimum shape for the hole, i.e.,

\footnotetext{
${ }^{9}$ By using nodal constraints within TOSCA.shape it would be possible to preserve the circular shape of the hole if desired, for example, for manufacturing purposes
} 


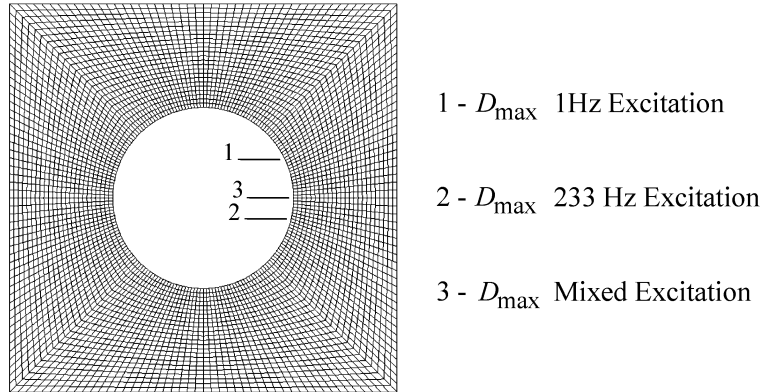

Fig. 11 Location of maximum damage, start design

the shape that minimizes damage to the hole's border, differs significantly among the three models. Geometry (a) is the result from the dynamic simulation with a $1 \mathrm{~Hz}$ excitation. The maximum damage appears at the same position as the maximum equivalent stress of a static deflection (see Fig. 9). As a consequence, the local surface curvature is lowered in this area by the optimizer.

Similar observations can be made for the second model, namely the $233-\mathrm{Hz}$ excitation. Here the maximum damage appears in the vicinity of the maximum equivalent stress of the modal stress tensor associated to eigenmode three. Therefore, the resulting shape of the hole (b) differs substantially from the prior example.

The resulting geometry of model three (c) with a superposition of the two excitations can be interpreted as a compromise between the two preceding shape results. Again, the location of the maximum damage shifts, this time in between the locations of the two other cases.

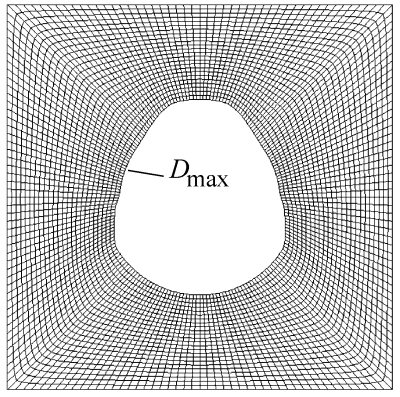

(a)

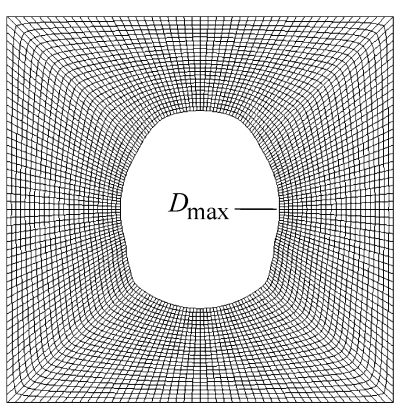

(c)

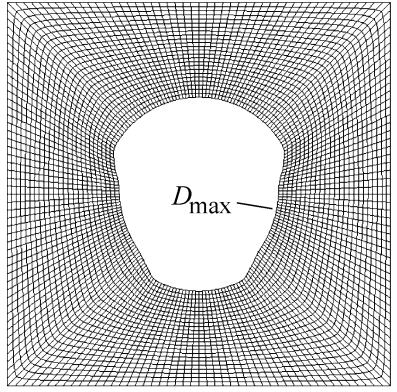

(b)
Fig. 12 Optimization results (shape) and location of maximum damage

\section{4 .2}

\section{Damage distribution}

For a quantitative analysis of the resulting damage distribution on the border of the hole, an analysis path of nodes along this border has been defined. In the following, nodal damage results for these nodes have been assessed. Figures 13-15 show the nodal damage distribution along the analysis path. For each of the three cases discussed above, the results are presented for the start design (circular hole) and the optimized design. In all three cases, the maximum occurring damage value has been reduced significantly by the optimization. Furthermore, it can be observed that the spatial extent of the damaged area increases. The appearance of two separate damaged areas in all three optimized designs can be explained by the fact that the border curvature of each of the optimized holedesigns is no longer concave. This effect would possibly have vanished if further iterations had been carried out.

Table 6 presents the values for the reduction of the maximum damage. Values are expressed in relative terms,

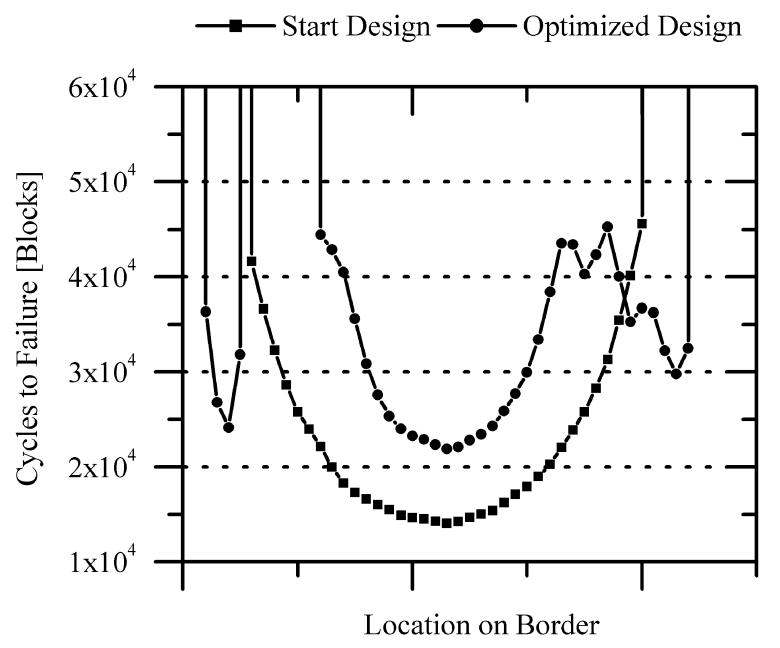

Fig. 13 Damage along the hole border $-1 \mathrm{~Hz}$ excitation

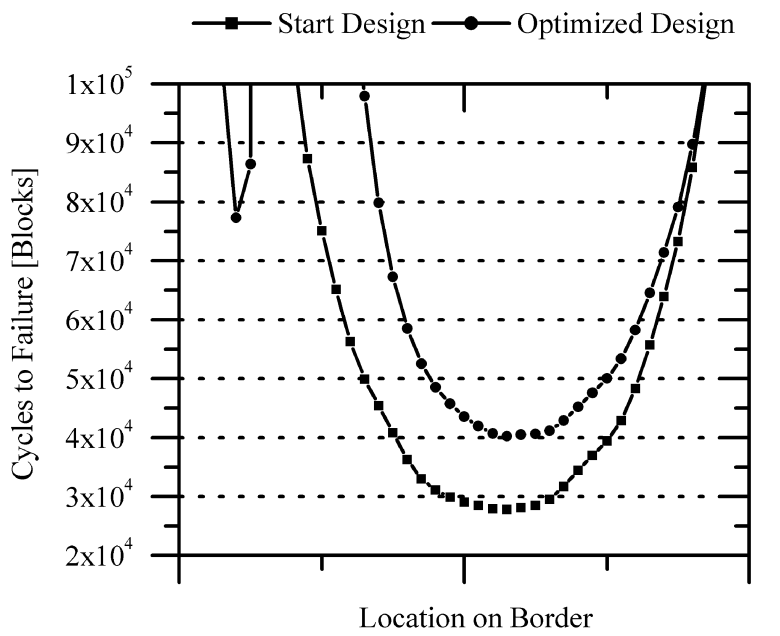

Fig. 14 Damage along the hole border $-233 \mathrm{~Hz}$ excitation 


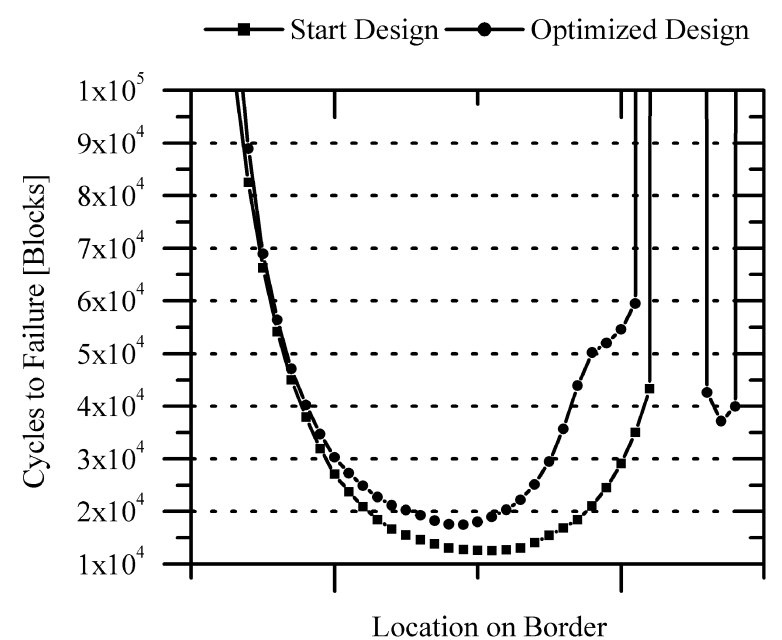

Fig. 15 Damage along the hole border - mixed excitation

Table 6 Reduction of the maximum damage

\begin{tabular}{lc}
\hline Model & Damage reduction [\%] \\
\hline \hline 1 Hz excitation & 56 \\
233 Hz excitation & 39 \\
Mixed excitation & 45 \\
\hline
\end{tabular}

i.e., as a percentage of the damage value of the respective start design. All achieved damage reductions are in the same order of magnitude.

As termination criterion, a limited number of iterations (eight) was defined to reduce computation times. This was justified because earlier investigations showed that the major damage reduction typically occurs within the first few iterations. Nevertheless, it is possible that an even better result could have been obtained with further iterations. The optimization process was stable and no convergence difficulties could be observed. However, convergence in the optimality criteria approach in TOSCA.shape does not mean convergence in a mathematical sense. In order to avoid a specified number of iterations, alternative termination criteria could have been used, for example, based on the relative change of the design response:

\section{"Terminate the optimization process if $\Delta \hat{D}_{\mathrm{Resp}} \leq \epsilon$ within the design area,"}

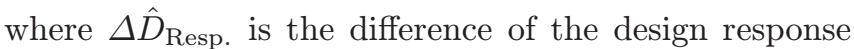
maxima of two successive iterations and $\epsilon$ is a given threshold.

\section{6}

\section{Concluding remarks}

The process for the durability-based shape optimization of dynamically loaded structural components as presented in this paper has proven feasible and applicable in the example discussed above. The importance of considering fatigue issues in the design process of such components can be clearly deduced from the plate example. A simple load scenario with a constant uniaxial load direction leads to significant differences in the optimum design. This is due to the different excitation frequencies that give rise to different excitation states of the plate. Summarizing, it can be stated that the optimum shape of a randomly loaded structural component depends on the frequency content of the emerging excitations. Shape optimization based on static stress results may not be sufficient in such cases since it is the accumulated local damage that determines the optimum shape rather than peak stresses of single load cases.

The basic approach and the implementation of the process allow its application for large models with complex mechanical systems and multi-axial random load scenarios. Many stand alone models for MSC.ADAMS, FALANCS or even TOSCA.shape are already available at many companies in the industry. Therefore, it is possible to directly apply the coupled process in a straightforward manner. Within the framework of ELAnO the authors, together with TRW Automotive Global R\&D, are currently working on the optimization of a suspension arm that is embedded in a full vehicle model. First applications in the industry have been reported and are gaining momentum.

Acknowledgements This work was supported by the Bundesministerium für Bildung und Forschung.

\section{References}

Allinger, P.; Bakhtiary, N.; Müller, O.; Mulfinger, F.; Puchinger, M.; Sauter, J. 1996: A new approach for sizing, shape and topology optimization. SAE International, International Congress $\&$ Exposition, Detroit. SAE Trans. 105(5), 745-761. Detroit: American Technical Publishers

Bannantine, J. A.; Comer, J.J.; Handrock, J.L. 1990: Fundamentals of metal fatigue analysis. Upper Saddle River, NJ: Prentice-Hall

Bishop, N.W.M.; Sherratt, F. 2000: Finite element based fatigue calculations. Glasgow: NAFEMS

Craig, R.R.; Bampton, M.C.C. 1968: Coupling of substructures for dynamic analyses. AIAA J. 6(7), 1313-1319

Fanni, M. 1993: Formoptimierung dynamisch belasteter Bauteile. Dissertation, Fakultät für Maschinenbau, University of Karlsruhe

Grunwald, J.E. 1996: A fatigue model for shape optimization based on continuum damage mechanics. Dissertation, Fakultät für Maschinenbau, University of Karlsruhe

Häußler, P.; Emmrich, D.; Müller, O.; Ilzhöfer, B.; Albers, A. 2001: Automated topology optimization of flexible components in hybrid finite element multibody systems using ADAMS/Flex and MSC.Construct. 2001 ADAMS European Users' Conference, Berchtesgaden, Germany. Berchtesgaden, Germany: Mechanical Dynamics 
Hurty, W.C. 1965: Dynamic analysis of structural systems using component modes. AIAA J. 3(4), 678-685

Ilzhöfer, B.; Müller, O.; Häußler, P.; Allinger, P.; Albers, A. 2000: Optimization based on parameters from life time prediction. NAFEMS-Seminar: Betriebsfestigkeit, Lebensdauer, Wiesbaden, Germany. Wiesbaden, Germany: NAFEMS

Ilzhöfer, B.; Müller, O.; Häußler, P.; Allinger, P.; Albers, A. 2001: Shape optimization based on lifetime prediction measures. International Conference on Engineering Design, ICED 01, Glasgow. Glasgow: S. Culley

Karush, W. 1939: Minima of functions of several variables with inequalities as side conditions. Dissertation, Department of Mathematics, University of Chicago, Chicago

Kuhn, H.W.; Tucker, A.W. 1951: Nonlinear programming. In: Neyman, J. (ed.), Proceedings of the second berkeley symposium on mathematical statistics and probability, vol. 2. Berkeley: University of California Press, pp. 481-492

LMS International 2002: FALANCS theory manual. LMS International: 3001 Leuven, Belgium

Müller, O.; Albers, A.; Allinger, P.; Sauter, J. 1999: Topology optimization of large real world structures. NAFEMS World Congress '99, Newport, vol. 2. Newport, RI: NAFEMS, pp. $827-839$

Müller, O.; Albers, A.; Ilzhöfer, B.; Häußler, P. 1999: Multidisciplinary shape and topology optimization and its integration in the product development process for the effective development of competetive products. ICED 99 12th International Conference on Engineering Design, Munich, 2(26). Munich: U. Lindemann, pp. 655-660

Müller, O.; Häußler, P.; Lux, R.; Ilzhöfer, B.; Albers, A. 1999: Automated coupling of MDI/ADAMS and MSC.CONSTRUCT for the topology and shape optimization of flexible mechanical systems. 1999 International ADAMS Users' Conference, Berlin. Berlin: Mechanical Dynamics

Sauter, J. 1992: Eine neue Gestaltoptimierungsstrategie nach dem Vorbild biologischer Strukturen. ZAMM 72(6), $566-570$

Schaeffer, G. 2001: MSC.Nastran primer for linear analysis. MSC.Software: Santa Ana, CA 92707, USA

Schnack, E.; Weikl, W. 2000: A Review about structural optimization concerning fatigue. In: Horvath, I.; Medland, A.J.; Vergeest, J.S.M. (eds.) Tools and Methods of Competitive Engineering, 3rd International Symposium, Delft, The Netherlands. Delft, The Netherlands: Delft University Press, pp. $539-550$

Schnack, E. 1978: Ein Iterationsverfahren zur Optimierung von Kerboberflächen. VDI-Forschungsheft 589. Dusseldorf: VDI-Verlag

Shabana, A.A. 1998: Dynamics of multibody systems, 2nd edn. Cambridge: Cambridge University Press 